Biochimica et Biophysica Acta, 600 (1980) 620-624

(C) Elsevier/North-Holland Biomedical Press

BBA 78874

\title{
THE LIPIDIC PARTICLE AS AN INTERMEDIATE STRUCTURE IN MEMBRANE FUSION PROCESSES AND BILAYER TO HEXAGONAL $\mathrm{H}_{\mathrm{II}}$ TRANSITIONS
}

\author{
A.J. VERKLEIJ a,*, C.J.A. VAN ECHTELD ${ }^{\text {b }}$, W.J. GERRITSEN ${ }^{\text {b }}$, P.R. CULLIS ${ }^{c}$ \\ and B. DE KRUIJFF a \\ a Institute of Molecular Biology and ${ }^{\mathrm{b}}$ Biochemistry Department, Rijksuniversiteit te \\ Utrecht, Transitorium 3, Padualaan 8, Uithof, Utrecht (The Netherlands) and \\ c Department of Biochemistry, University of British Columbia, Vancouver, BC V6T 1 W5 \\ (Canada)
}

(Received February 19th, 1980)

Key words: Cardiolipin; Cholesterol; Fusion process, Phosphatidylcholine; Phosphatidylethanolamine; (Freeze-fracture, Liposome)

\section{Summary}

Small unilamellar vesicles comprised of a mixture of phosphatidylethanolamine/phosphatidylcholine/cholesterol $(3: 1: 2)$ fuse to form large multilamellar vesicles on increasing the temperature from 0 to $50^{\circ} \mathrm{C}$. This event is associated with the appearance of lipidic particles at the fusion sites, consistent with a role as intermediary structures during the fusion process. Further, for phosphatidylcholine/cardiolipin $(1: 1)$ liposomes in the presence of $\mathrm{Mn}^{2+}$ a direct relationship between lipidic particles and the hexagonal $\left(\mathrm{H}_{\mathrm{II}}\right)$ phase is demonstrated which suggests that lipidic particles can also occur as intermediaries between bilayer and hexagonal $\left(\mathrm{H}_{\mathrm{II}}\right)$ structures.

Individual lipid species isolated from biological membranes commonly assume the bilayer or hexagonal $\left(\mathrm{H}_{\mathrm{II}}\right)$ arrangements on dispersal in excess water $[1,2]$. As noted in earlier reports, mixtures of 'bilayer' lipids with hexagonal ' $\mathrm{H}_{\text {II }}$ ' phase lipids, including the total lipid extracts of various biological membranes, can exhibit a new organization known as the 'lipidic particle' [2-4] which has been suggested to reflect the presence of intra-bilayer inverted micelles $[3,4]$. This structure, visualized as particles and pits in freeze-fracture micrographs, is also accompanied by the appearance of a narrow, symmetric ${ }^{31} \mathrm{P}$ NMR component indicative of isotropic motional averaging $[2,4]$. Two points

* On leave at the Biological Laboratories, Harvard University, 16 Divinity Avenue, Cambridge, Massachusetts 02138 , U.S.A. 
of interest concerning lipidic particles are that they occur at the fusion interface during $\mathrm{Ca}^{2+}$-induced fusion of cardiolipin-phosphatidylcholine vesicles [5], and that narrow symmetrical ${ }^{31} \mathrm{P}-\mathrm{NMR}$ components are often observed as intermediaries between bilayer and hexagonal $\left(\mathrm{H}_{\mathrm{II}}\right)$ phases [2,4]. It is therefore of interest to determine whether formation of lipidic particles is a common intermediary feature of fusion processes, and whether such structures can also act as intermediaries in bilayer- $\mathrm{H}_{\mathrm{II}}$ transitions. In the present work we show that temperature-induced fusion of unilamellar phosphatidylethanolamine/phosphatidylcholine/cholesterol vesicles is accompanied by the appearance of lipidic particles at sites of fusion and that organized rows of these particles appear to occur as precursors to the cylindrical organization of $\mathrm{H}_{\mathrm{II}}$ phase lipids. These observations support the possibility that lipidic particles act as intermediaries during fusion events in general, as well as the proposal that lipidic particles reflect the presence of inverted micellar structures.

Fusion experiments were performed on mixtures of 1,2-dioleoyl-sn-glycero3-phosphorylethanolamine $\left(18: 1_{c} / 18: 1_{c}\right.$ phosphatidylethanolamine $) / 1,2$ dioleoyl-sn-glycero-3-phosphorylcholine $\left(18: 1_{c} / 18: 1_{c}\right.$ phosphatidylcholine)/ cholesterol in the molar proportions $3: 1: 2$. This material was dispersed in $100 \mathrm{mM} \mathrm{NaCl}, 10 \mathrm{mM}$ Tris-HCl (pH 7.0) by vortex mixing to arrive at a $10 \mathrm{mM}$ phospholipid concentration. Subsequently this material was sonicated intermittently ( $30 \mathrm{~s}$ sonication, $1 \mathrm{~min}$ waiting, employing a Branson tip sonicator, power setting 5 ) for a total sonication time of $3 \mathrm{~min}$ to give a transparent vesicle dispersion. Freeze-fracture micrographs obtained from this material reveal the presence of predominantly unilamellar vesicles with smooth fracture faces and diameters ranging from 300 to $2000 \AA$ (Fig. 1A).

Freeze-fracture studies of unsonicated dispersions of this lipid mixture have revealed bilayer structure if the material is kept at low (e.g., $0 \leqslant t \leqslant 10^{\circ} \mathrm{C}$ ) temperatures [4]. However, a brief $(10 \mathrm{~min})$ incubation at $50^{\circ} \mathrm{C}$ results in the appearance of regions of hexagonal $\left(\mathrm{H}_{\mathrm{II}}\right)$ phase and lipidic particles [4]. It was therefore reasoned that if lipidic particles participate as intermediaries in fusion events, enhanced fusion of the sonicated vesicles should be observed on incubation at $50^{\circ} \mathrm{C}$. That this is the case is revealed in the freeze-fracture micrograph of Fig. 1B where a dramatic increase in the size of the vesicles is observed as the incubation time increases. This was accompanied by an increasingly milky appearance of the vesicle suspension. It may be noted that lipidic particles are present, observed as complementary particles and pits on the concave and convex fracture faces, respectively. The average diameter of the particles is approx. $110 \AA$, similar to those observed for the corresponding nonsonicated dispersions [4]. This distribution of lipidic particles closely parallels that observed for cardiolipid-phosphatidylcholine vesicles where fusion was induced by $\mathrm{Ca}^{2+}[5]$ in that the particles are predominantly found on vesicles which have an unusual dimpled appearance, and appear to be preferentially located in regions corresponding to the fusion interface. Furthermore, this would suggest that the appearance of lipidic particles in the non-sonicated liposomes of phosphatidylethanolamine/phosphatidylcholine/cholesterol $(3: 2: 1)$ may be associated with fusion either between or within these multilayered structures.

The occurrence of lipidic particles after incubation at $50^{\circ} \mathrm{C}$ may be attrib- 


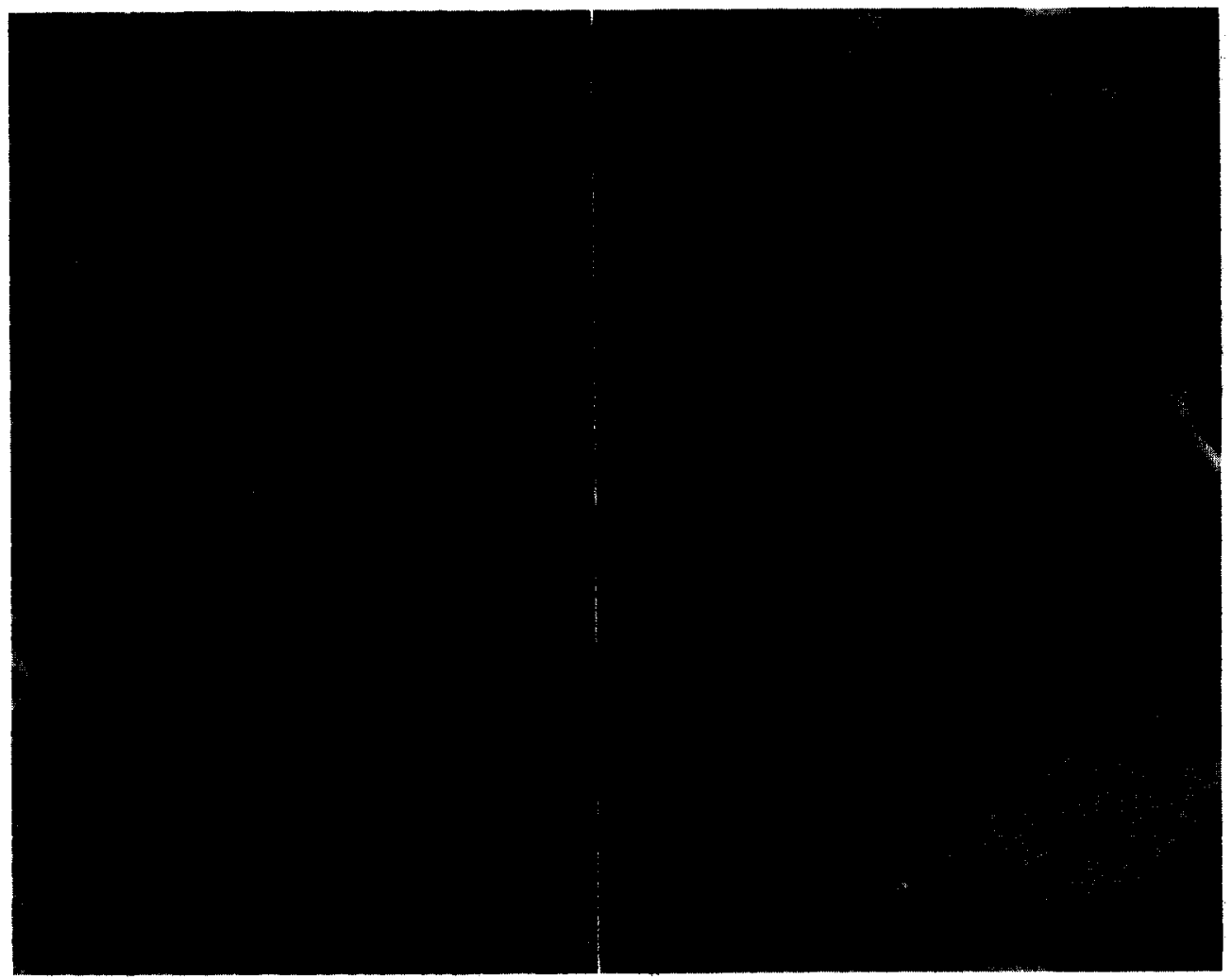

Fig. 1. Freeze-fracture micrographs of a mixture of $18: 1_{c} / 18: 1_{c}$ phosphatidylethanolamine/18: $1_{c} /$ $18: 1_{c}$ phosphatidylcholine/cholesterol at a molar ratio of $3: 1: 2$, sonicated at $0^{\circ} \mathrm{C}$ (A) and after 10 min incubation at $50^{\circ} \mathrm{C}$ and quenched from $4^{\circ} \mathrm{C}$ (B). Magnification $\times 93000$. Freeze-fracturing has been performed according to the standard procedures. Gly cerol was added to prevent freeze-damage.

uted to the preference of the phosphatidylethanolamine component for the $\mathrm{H}_{\mathrm{II}}$ phase at these temperatures [6]. This parallels the situation in the cardiolipidphosphatidylcholine vesicles, where the presence of $\mathrm{Ca}^{2+}$ converts the cardiolipin to an $\mathrm{H}_{\mathrm{II}}$-preferring species. It may be noted that a similar situation can be obtained in lipid mixtures containing phosphatidylserine and phosphatidylethanolamine, where the presence of $\mathrm{Ca}^{2+}$ can allow this preference of the phosphatidylethanolamine for the $\mathrm{H}_{\text {II }}$ phase to be expressed [7,8], again potentially leading to formation of lipidic particles. We suggest that these structures (interpreted as inverted micelles) may be an intermediary (see model of Ref. 9) in fusion events in general.

In a previous work we have suggested that lipidic particles reflect the presence of intra-bilayer inverted micellar lipid structures [3,4]. This hypothesis was based largely on the observations that mixtures of 'bilayer' lipids with lipids preferring the 'inverted' (i.e., polar head groups oriented inwards towards an aqueous channel) $H_{\text {II }}$ phase give rise to such structures [4]. In order to place this possibility on a more sound foundation, and also with a view towards understanding the mechanism of the bilayer $-\mathrm{H}_{\mathrm{II}}$ transition more clearly, we re-investigated the behaviour of cardiolipin-egg yolk phosphatidylcholine 
(1:1) dispersions in the presence of $\mathrm{Ca}^{2+}$ and $\mathrm{Mn}^{2+}$, employing freeze-fracture. $\mathrm{Mn}^{2+}$ occurs in relatively high concentrations in mitochondria [9] and can exert an effect comparable to $\mathrm{Ca}^{2+}$ on cardiolipin-phosphatidylcholine dispersions (Verkleij, A.J., unpublished results). The aim of these experiments was to try to 'capture' events corresponding to a transition between lipidic particles and the inverted lipid cylinders of the $\mathrm{H}_{\mathrm{II}}$ phase which are both present after addition of divalent cations to dispersions of these lipids.

Occasionally one can observe interesting transitory stages from lipidic particles to hexagonal ${ }_{I I}$ phases as shown in Fig. 2. There are three major points of interest. Firstly, two types of structure corresponding to an inverted cylinder $\left(\mathrm{H}_{\mathrm{II}}\right.$ phase $)$ lipid organization are present. One of these has a diameter of $100 \AA$, which is similar to the diameter of the lipidic particles, whereas the other has a

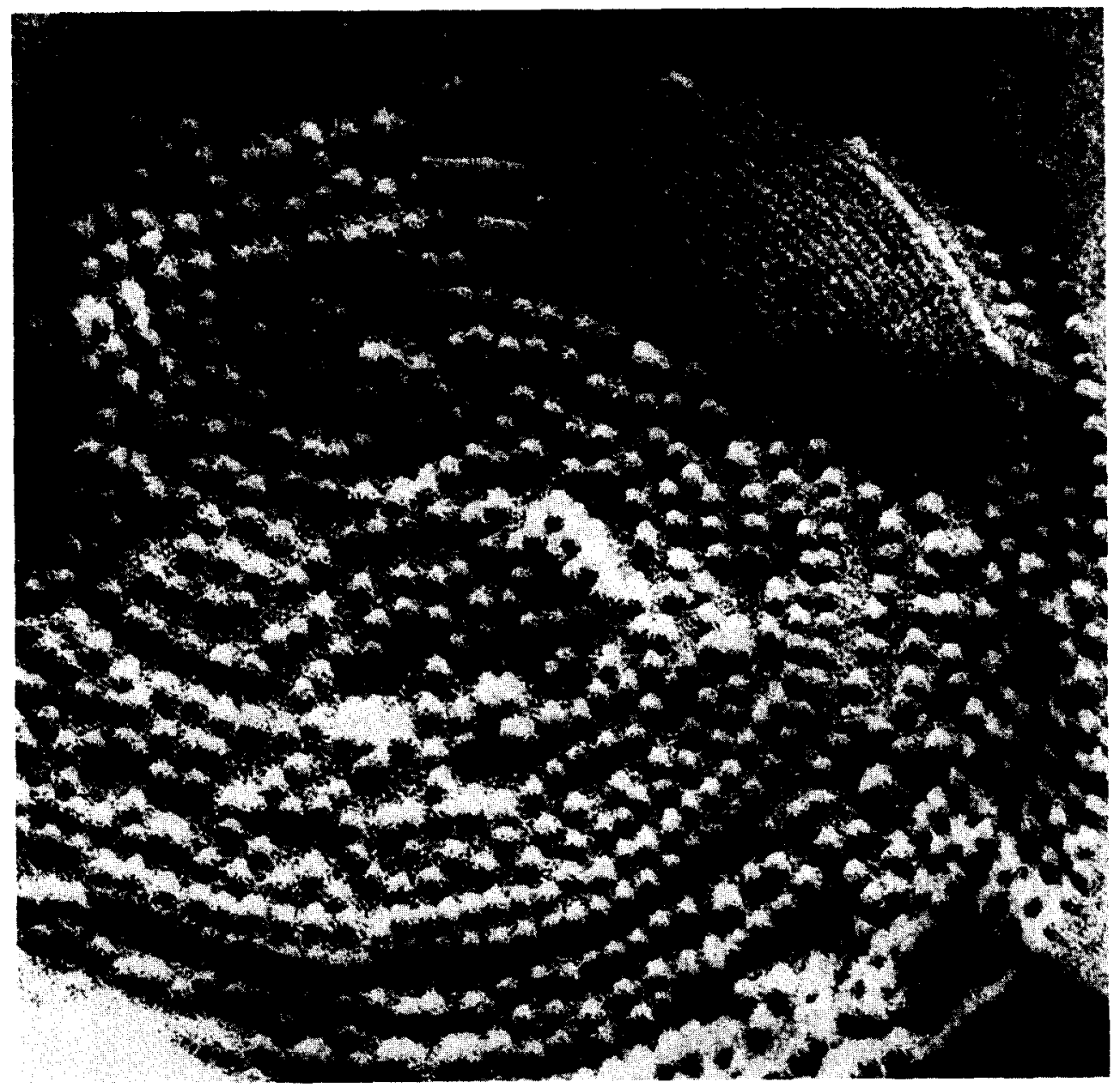

Fig. 2. Freeze-fracture micrograph of a mixture of dioleoylphosphatidylcholine/cardiolipid (from bovine heart from Sigma) at a ratio of $1: 1$, prepared by the Triton method as described in Ref. 5 in the presence of $2 \mathrm{mM} \mathrm{MnCl}$. Lipid concentration is $2 \mathrm{mM}$ giving a $\mathrm{Mn}^{2+} /$ cardiolipin ratio of 2 . Note hexagonal ( $\mathrm{H}_{\mathrm{II}}$ ) cylinders with a diameter of about $100 \AA$ at the transition from particles to hexagonal (H $\mathrm{H}_{\mathrm{II}}$ ) cylinders with a diameter of 60 A. Magnification $\times 200000$. 
diameter of $60 \AA$ corresponding to the diameter of pure cardiolipin- $\mathrm{Ca}^{2+}$ hexagonal $H_{\text {II }}$ cylinders [11]. Secondly, the cylinders of the $H_{\text {II }}$ phase lipids are curved, indicating a certain degree of flexibility. Finally, and more instructively, lipidic particles appear in highly organized rows extending from the $100 \AA$ diameter inverted cylinders. These observations are clearly consistent with an interpretation of lipidic particles as inverted micelles, which can fuse to form the inverted cylinders of the $\mathrm{H}_{\mathrm{II}}$ phase, thus acting as intermediaries in the bilayer to hexagonal $\left(\mathrm{H}_{\mathrm{II}}\right)$ transition. Further, the observation of the $60 \AA$ diameter hexagonal phase cylinders suggests that segregation of cardiolipindivalent cation complex and phosphatidylcholine can occur subsequently.

In summary, the results presented here provide clear evidence for the occurrance of lipidic particles as intermediary structures in membrane fusion processes and bilayer-to-hexagonal $\left(\mathrm{H}_{\mathrm{II}}\right)$ phase transitions, and support the hypothesis that lipidic particles reflect the presence of inverted micelles.

\section{Acknowledgements}

We would like to thank J. Leunissen-Bijvelt for technical assistance. A.J.V. is in part supported by a stipend of the Netherlands Organization for the Advancement of Pure Research (Z.W.O.).

\section{References}

1 Luzatti, V., Gulik-Krzywicki, T. and Tardieu, A. (1968) Nature 218, 1031-1034

2 Cullis, P.R. and de Kruijf, B. (1979) Biochim. Biophys. Acta B59, 399-420

3 Verkleit, A.J., Mombers, C., Leunissen-Bljvelt, J. and Ververgaert, P.H.J.T. (1979) Nature 279, 162163

4 De Kruijf, B., Vexkleij, A.J., van Echteld, C.J.A., Gerritsen, W.J., Mombers, C., Noordam, P.C. and de Gier, J. (1979) Biochim. Biophys. Acta 555, 200-209

5 Verkleij, A.J., Mombers, C., Gerritsen, W.J., Leunissen-Bijvelt, J, and Cullis, P.R. (1979) Blochim. Biophys. Acta 555, 358-361

6 Cullis, P.R., van Dijck, P.W.M., de Kruijff, B. and de Gier, J. (1978) Biochim. Biophys. Acta 513, 2130

7 Cullis, P.R. and Verklej, A.J. (1979) Biochim. Biophy s. Acta 552, 546-551

8 Hope, M.J. and Cullis, P.R. (1879) FEBS Lett. 107, 323-327

9 Cullis, P.R. and Hope, M.J. (1978) Nature 271, 672-674

10 Ogawa, S., Rottenberg, H., Brown, T.R., Schulman, R.G., Costillo, C.L. and Glynn, P. (1979) Proc. Natl. Acad. Sci. U.S.A. 76, 1796-1800

11 Deamer, D.W., Leonard, R., Tardieu, A. and Branton, D. (1970) Biochim. Biophys. Acta 219, 47-60 